\title{
Introduction to the Book, Policy and Initiative Reviews Section
}

\section{Meg Holden ${ }^{1}$}

Published online: 21 September 2018

(C) Springer Nature Switzerland AG 2018

The International Journal of Community Well-Being is being launched at what we predict will be a take-off point for research, policy and practice in the wide-ranging field of well-being. This, of course, makes it a good time to launch the journal, and so to provide a platform to convene and support and spur forward the burgeoning field. At the same time, the flourishing of well-being is not occurring from a single central source point, but many. We are not at this launch point because major research universities have established new units to focus on community well-being - although we applaud and appreciate the work being done in places like Cambridge University's Well-Being Institute, McGill University's Institute for Human Development and Wellbeing, and other scholarly units where the study of well-being is rising in prominence. We are not at this point because of a single flagship launch of major new government or non-government funding programs or initiatives, although we think the work of the UK's new Minister of Loneliness, France's 2018 International Forum for Well-Being, and the OECD's How's Life? Initiative, among many others, are all worth our attention. Nor should we fail to notice trends toward more funding and partnership opportunities in the area of well-being emerging from government ministries of health, veterans' services, youth and more, local governments, philanthropies, and other institutions.

It is more the case that, when we take the growing sum of events and efforts like these into account, we cannot help but perceive that we find ourselves in the worst of times, as well as the best of times. By this, we mean that we notice the following conflicting and contradictory trends:

- So many of us are suffering, in ways that are poorly understood, from scarcities of key assets and essentials of community well-being;

- So many of us are setting higher aspirations for our own individual and group abilities to control our physical and mental health, to improve our own lives and thrive under difficult conditions;

Meg Holden

mholden@sfu.ca

1 Urban Studies and Geography, Simon Fraser University, Burnaby, BC, Canada 
- So many of us are hitting new lows in the sense of trust we have in our governments and our neighbours and the control we feel we have over decisions and actions being taken all around us, that are not serving our interests or those of communities we care about;

- So many of us are heartened by a sense of gaining new leverage in debate from new findings of connections among forces that appear disparate but that we know are connected to community well-being, from urban design, to health, to economic development, to crime and marginalization, to sociability, and beyond;

- So many of us feel a keen sense of defeat when considering our societal failure to meet the grand goals of the previous generation, such as the Kyoto Protocol (of the United Nations Framework Convention on Climate Change) and the United Nations Millennium Development Goals, and are skeptical of the seriousness of such efforts to mobilize leaders across big cultural divides to reach shared goals;

- So many of us feel motivated by the striking of global commitments to meet new grand societal goals, such as the Paris Climate Agreement and the United Nations Sustainable Development Goals; and feel compelled to push for the kind of common purpose that such goals generate in other areas of global significance, such as the treatment of refugees and displaced persons, religious freedom, and freedom from sexual and racial violence.

All of these trends are pulling our field in new directions, and pulling new people and energy into the orbit of our field to study and practice. As daunting as this embarrassment of hopes and fears, failures and possibilities may be, there is no alternative to reading and thinking more, reinforcing habits of critique and dialogue, working to integrate the lessons of our actions into reflections, and conversely, to mobilize our knowledge into action. This is the purpose we have established for this reviews section. We seek more than book reviews here, although naturally we are always on the lookout for reviews of new books that offer insight into our thinking. Beyond traditional books, we also intend to feature reviews of other major new directions in the community well-being field, whether these take the form of new policies, plans, major reports, or documented initiatives. From disciplines of psychology to philosophy, management to sociology to human ecology - and, we really hope, spaces in between. New initiatives we would like to know about might be in practices of public health or urban design, sustainability or resilience, tourism or recidivism.

We hope this reviews section can serve as a touchstone for all kinds of documented, significant new work in the field of community well-being. We are also open to comparative reviews. Please contact the special reviews section editor, Dr. Meg Holden, at mholden@sfu.ca with your idea for a review. And please add this new section of the International Journal of Community Well-Being to your repertoire of sources of strong, constructive critique that reveals and helps chart the direction of our field of study and practice. 